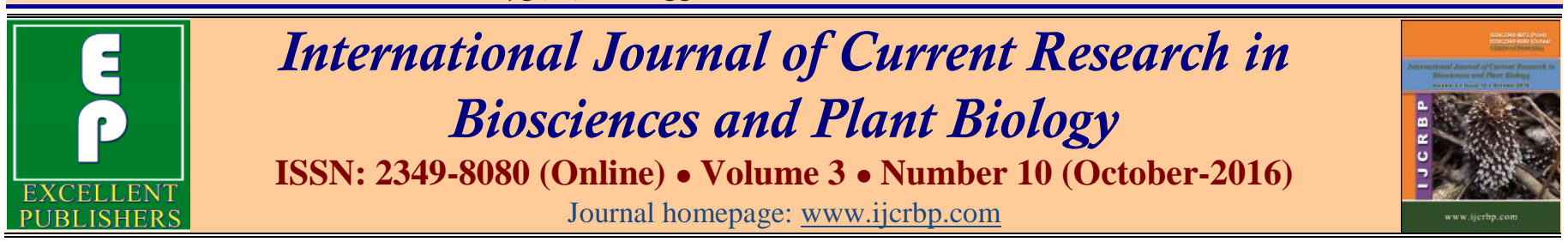

\title{
Studies on Characters Association and Path Coefficient Analysis for Yield and Water Use Efficiency Traits in Groundnut (Arachis hypogaea L.)
}

\author{
K. John* and P. Raghava Reddy \\ Regional Agricultural Research Station, Institute of Frontier Technology, Tirupati-517502, India \\ *Corresponding author.
}

\begin{abstract}
The estimate of genotypic correlation coefficients in general is higher than their corresponding phenotypic correlations indicating strong inherent association among the traits. Pod yield had significant positive association with SPAD chlorophyll meter reading at 60 days after sowing, harvest index, non significant positive association with SPAD chlorophyll meter reading at 40 days after sowing, days to maturity, specific leaf area at 60 days after sowing, relative water content at 40 days after sowing and dry haulms yield per plant. These characters can be considered as criteria for selection for higher yield, as these were mutually and directly associated with pod yield. Path coefficient analysis revealed that, the maximum positive direct positive effect on pod yield per plant was contributed by days to maturity, SPAD chlorophyll meter reading at 40 days after sowing and 60 days after sowing, specific leaf area at 60 days after sowing, specific leaf area at 40 days after sowing and 60 days after sowing, dry haulms yield per plant and harvest indicating that these traits are the important yield contributing characters.
\end{abstract}

\section{Article Info}

Accepted: 21 September 2016

Available Online: 06 October 2016

\section{Ke ywords}

Characters association

Groundnut

Path coefficient analysis

Water use efficiency traits

\section{Introduction}

Groundnut is the important oilseed crop of India. Though it leads in area and production in the world its productivity is low due to various abiotic and biotic stresses. Further, pod yield besides physiological traits in groundnut are quantitatively inherited complex traits and is highly influenced by environment. Understanding the relationships among yield and yield components is of paramount importance for making the best use of these relationships in selection. The correlation coefficient may be confounded with indirect effect due to common association inherent in trait interrelationships. Therefore information derived from the correlation coefficients can be augmented by partitioning correlations into direct and indirect effects by path coefficient analysis. In order to achieve the goal of increased production by increasing the yield potential of crop, knowledge of direction and magnitude of association between various traits is essential for plant breeders. Accordingly, the present investigation was aimed to study the association of pod yield and its component traits in elite groundnut genotypes.

\section{Materials and methods}

Twenty four $F_{1} s$ along with six lines and four testers were sown in a Randomised Block Design (RBD) with three replications during kharif 2014. Each parent was sown in 3 rows of $3 \mathrm{~m}$ length while $F_{1} s$ were raised in a 
single row of $3 \mathrm{~m}$ length with a spacing of $22.5 \times 10 \mathrm{~cm}$. All the 24 treatments along with parents were allotted at random to the experimental plots in each replication. Thirty random plants per replication were sampled for recording observations from each cross, while ten random plants were taken from each parent in each replication and their mean values were used. The experiment was conducted in a red sandy loam soil with a neutral $\mathrm{pH}$, low in organic carbon. Recommended agronomic and plant protection measures were adopted for the conduct of experiment.

The data were recorded for viz., days to 50 per cent flowering, days to maturity, SPAD chlorophyll meter reading at 40 DAS and 60 DAS, specific leaf area at 40 DAS and 60 DAS, specific leaf weight at 40 DAS and 60 DAS, relative water content 40 DAS and 60 DAS, root length $(\mathrm{cm})$, dry haulms weight per plant $(\mathrm{g})$, harvest index $(\%)$ and pod yield per plant $(\mathrm{g})$. The water use efficiency traits were measured on all four- leaflets of third leaf from the top on main axis at 40 and 60 DAS. Genotypic and phenotypic correlation coefficients were calculated among the genotypes using the formulae suggested by Al-Jibouri et al. (1958). Path coefficient analysis was carried out by using phenotypic and genotypic correlation coefficients as per the method suggested by Dewey and Lu (1959).

\section{Results and discussion}

The genotypic and phenotypic correlations are presented in Table 1. In general, the values of genotypic correlations were higher than their phenotypic correlations in the present investigation. This indicated that though there was high degree of association between two variables at genotypic levels, its phenotypic expression was deflated by the influence of environment. In the present study, pod yield per plant had significant positive association with SPAD chlorophyll meter reading at 60 days after sowing, harvest index, non significant positive association with SPAD chlorophyll meter reading at 40 days after sowing, days to maturity, specific leaf area at 60 days after sowing, relative water content at 40 days after sowing and dry haulms yield per plant, but nonsignificant negative association with specific leaf area at 40 days after sowing. These characters can be considered as criteria for selection for higher yield, as these were mutually and directly associated with pod yield. Similar results were obtained by John et al. (2012) and Vaithiyalingan et al. (2010) reported positive association of pod yield with harvest index.
The association of days to 50 per cent flowering with days to maturity, SPAD chlorophyll meter reading at 60 days after sowing and specific leaf weight at 60 days after sowing was found to be significant and positive non significant association with SPAD chlorophyll meter reading at 40 days after sowing, specific leaf weight at 40 days after sowing, relative water content at 40 days after sowing, dry haulms yield per plant and harvest index. This character had show negative non significant association with specific leaf area at 40 days after sowing, relative water content at 60 days after sowing, root length and pod yield per plant.

Days to maturity possessed significant and positive association with relative water content at 40 days after sowing and harvest index and positive non significant association with SPAD chlorophyll meter reading both at 40 days after sowing and 60 days after sowing, specific leaf area 40 days after sowing, specific leaf weight 60 days after sowing, relative water content at 60 days after sowing, dry haulms yield per plant and pod yield per plant. But negative non significant correlation with specific leaf area at 60 days after sowing, specific leaf weight at 40 days after sowing and root length. John et al. (2012) reported positive association of days to maturity with dry haulm yield per plant.

SPAD chlorophyll meter reading at 40 days after sowing established significant positive association with SPAD chlorophyll meter reading at 60 days after sowing and negative significant association with dry haulms yield per plant. It had shown positive non significant association with specific leaf area at 40 days after sowing, relative water content at 40 days after sowing, and pod yield per plant and negative non significant correlation with specific leaf area at 60 days after sowing, specific leaf area at 40 days after sowing and 60 days after sowing, relative water content at 60 days after sowing and harvest index.

The relationship of SPAD chlorophyll meter reading at 60 days after sowing with Relative water content SPAD chlorophyll meter reading at 60 days after sowing was significant and positive, while its association with other characters viz., specific leaf area SPAD chlorophyll meter reading at 40 days after sowing, specific leaf weight SPAD chlorophyll meter reading at 60 days after sowing, root length, dry haulms yield per plant, harvest index and pod yield per plant was found to be positive but non-significant, whereas it's correlation with specific leaf area at 60 days after sowing, specific leaf weight at 40 days after 
sowing, relative water content at 40 days after sowing was negative and non significant.

The relationship of specific leaf area at 40 days after sowing with specific leaf area at 60 days after sowing, specific leaf weight at 60 days after sowing and dry haulms yield per plant was positive and non significant, but its association with specific leaf weight at 40 days after sowing, relative water content at 40 days after sowing and 60 days after sowing, root length, harvest index and pod yield per plant was negative and non significant.

Specific leaf area at 60 days after sowing possessed significant and positive association with relative water content at 60 days after sowing and negative significant association with specific leaf weight at 40 days after sowing and harvest index, but non significant positive association with pod yield per plant and negative non significant correlation with specific leaf area at 60 days after sowing, relative water content at 40 days after sowing, root length and dry haulms yield per plant. Earlier John et al. (2012) reported that specific leaf area established negative association with specific leaf weight.

Leaves with high SLW (inverse of SLA) were cooler under a given radiation load due to higher stomatal conductance resulting in more evaporative cooling. SLA was low in cooler climate (night temperature low), greater deposition of starch and other non-structural carbohydrates in the chloroplasts (Araus et al., 1989). Based on many experiments, over a wide range of environments and cultivars, it was concluded that SLA was closely and negatively correlated with WUE and positively correlated with carbon isotope discrimination. This highlights the possibility of using SLA as a more rapid and inexpensive technique for selection of WUE (Nageswara Rao and Wright, 1994).

The association of specific leaf weight at 40 days after sowing with specific leaf weight at 60 days after sowing and harvest index showed significant positive association and significant negative association with dry haulms yield per plant. Its association with pod yield per plant was non-significant and negative, whereas it showed a non significant positive association with relative water content at 40 days after sowing and 60 days after sowing and root length.

Specific leaf weight at 60 days after sowing with relative water content at 60 days after sowing showed significant positive association and non significant positive relationship with root length and harvest index, where as its association with relative water content at 40 days after sowing, dry haulms yield per plant and pod yield per plant was negative and non significant.

The relationship of relative water content at 40 days after sowing with harvest index was significant and positive and with relative water content at 60 days after sowing, dry haulms yield per plant and pod yield per plant was positive and non significant. Its relationship with root length was significant negative association. Relative water content at 60 days after sowing with harvest index was significant and positive and with root length was positive and non significant, but with dry haulms yield per plant was negative significant association. Its relationship with pod yield per plant was non-significant negative association.

Root length established a significant and positive relationship with dry haulms yield per plant and harvest index and negative non significant association with pod yield per plant. The relationship of dry haulms yield per plant with harvest index was positive and significant. Harvest index had significant positive association with pod yield per plant.

From the present studies, it is evident that pod yield had significant positive association with SPAD chlorophyll meter reading at 60 days after sowing, harvest index, non significant positive association with SPAD chlorophyll meter reading at 40 days after sowing, days to maturity, specific leaf area at 60 days after sowing, relative water content at 40 days after sowing and dry haulms yield per plant. These characters can be considered as criteria for selection for higher yield, as these were mutually and directly associated with pod yield.

Pod yield is a complex dependent character and is contributed by several components. Correlation studies simply measures the association of yield and yield attributes and does not give the actual dependence of yield on the correlated characters. Path coefficient analysis is an effective method to determine the direct and indirect causes of associations and also permits to examine the specific forces acting to produce to a given correlation. Hence, an attempt was made to study the direct and indirect effects of characters on pod yield through path coefficient analysis in twenty four cross combinations in groundnut. The phenotypic path coefficients worked-out for yield and its components in $\mathrm{F}_{1}$ generation are furnished in Table 2 . 
Table 1. Phenotypic $(P)$ and genotypic $(G)$ correlation coefficients among water use efficiency traits and pod in groundnut

\begin{tabular}{|c|c|c|c|c|c|c|c|c|c|c|c|c|c|c|c|}
\hline \multirow[t]{2}{*}{ Character } & & \multirow{2}{*}{$\begin{array}{l}\text { Days to } \\
\mathbf{5 0 \%} \\
\text { flowering }\end{array}$} & \multirow{2}{*}{$\begin{array}{l}\text { Days to } \\
\text { maturit } \\
y\end{array}$} & \multicolumn{2}{|c|}{$\begin{array}{l}\text { SPAD chlorophyll } \\
\text { meter reading at }\end{array}$} & \multicolumn{2}{|c|}{$\begin{array}{l}\text { Specific leaf area } \\
\left(\mathrm{cm}^{2} \mathrm{~g}^{-1}\right) \text { at }\end{array}$} & \multicolumn{2}{|c|}{$\begin{array}{l}\text { Specific leaf weight } \\
\left(\mathrm{g} \mathrm{cm}^{-2}\right) \text { at }\end{array}$} & \multicolumn{2}{|c|}{$\begin{array}{l}\text { Relative water } \\
\text { content (\%) at }\end{array}$} & \multirow{2}{*}{$\begin{array}{l}\text { Root } \\
\text { length } \\
\text { (cm) }\end{array}$} & \multirow{2}{*}{$\begin{array}{l}\text { Dry haulm } \\
\text { weight per } \\
\text { plant }(\mathrm{g})\end{array}$} & \multirow{2}{*}{$\begin{array}{l}\text { Harvest } \\
\text { index } \\
(\%)\end{array}$} & \multirow{2}{*}{$\begin{array}{l}\text { Pod yield } \\
\text { per plant } \\
\text { (g) }\end{array}$} \\
\hline & & & & 40 DAS & 60 DAS & 40 DAS & 60 DAS & 40 DAS & 60 DAS & 40 DAS & 60 DAS & & & & \\
\hline \multirow[t]{2}{*}{ Days to $50 \%$ flowering } & $P$ & 1.0000 & $0.2419^{*}$ & 0.2196 & $0.2801^{* *}$ & 0.0045 & -0.0712 & 0.0161 & $0.2530^{*}$ & 0.0078 & -0.0965 & -0.0752 & 0.1834 & 0.0891 & -0.2070 \\
\hline & G & 1.0000 & 1.2084 & 0.2460 & 0.3362 & 0.0079 & -0.0862 & 0.1286 & -0.5151 & 0.0345 & -0.1033 & -0.4688 & 0.1998 & 0.0979 & -0.3697 \\
\hline \multirow[t]{2}{*}{ Days to maturity } & $P$ & & 1.0000 & 0.1442 & 0.1010 & 0.1796 & -0.1398 & -0.1941 & 0.1106 & $0.7135^{* *}$ & 0.1222 & -0.0159 & 0.0819 & $0.2568^{* *}$ & 0.0699 \\
\hline & G & & 1.0000 & 0.3549 & 0.5538 & 0.8161 & -0.6214 & -2.2789 & 0.3422 & 3.4243 & 0.7055 & 0.4878 & 0.4198 & 1.2436 & 0.5758 \\
\hline \multirow{2}{*}{$\begin{array}{l}\text { SPAD chlorophyll meter reading at } 40 \\
\text { DAS }\end{array}$} & $P$ & & & 1.0000 & $0.3948^{* *}$ & 0.1530 & -0.1616 & -0.1709 & -0.0101 & 0.0200 & -0.1411 & -0.0562 & $0.4398^{* *}$ & -0.0713 & 0.0296 \\
\hline & G & & & 1.0000 & 0.4288 & 0.1617 & -0.1669 & -0.3435 & 0.1415 & -0.0052 & -0.1567 & -0.8648 & 0.457 & -0.0725 & 0.0181 \\
\hline \multirow{2}{*}{$\begin{array}{l}\text { SPAD chlorophyll meter reading at } 60 \\
\text { DAS }\end{array}$} & $P$ & & & & 1.0000 & 0.0922 & -0.1410 & -0.0236 & 0.1318 & -0.0890 & $-0.3240^{\star *}$ & 0.1640 & 0.0435 & 0.1275 & $0.2391^{*}$ \\
\hline & G & & & & 1.0000 & 0.0929 & -0.1471 & -0.0816 & -0.6115 & -0.1079 & -0.3712 & 0.7664 & 0.0493 & 0.1381 & -0.2132 \\
\hline \multirow[t]{2}{*}{ Specific leaf area $\left(\mathrm{cm}^{2} \mathrm{~g}^{-1}\right)$ at 40 DAS } & $P$ & & & & & 1.0000 & 0.1541 & $-0.7612^{* *}$ & $-0.2421^{*}$ & -0.1253 & -0.1879 & -0.0375 & 0.1151 & -0.1829 & -0.1168 \\
\hline & G & & & & & 1.0000 & 0.1578 & -1.5830 & 0.7027 & -0.1355 & -0.2057 & -0.2421 & 0.1164 & -0.1855 & -0.1622 \\
\hline \multirow[t]{2}{*}{ Specific leaf area $\left(\mathrm{cm}^{2} \mathrm{~g}^{-1}\right)$ at 60 DAS } & $P$ & & & & & & 1.0000 & $-0.4205^{\star *}$ & -0.1706 & -0.1698 & $0.3070^{* *}$ & -0.1887 & -0.0033 & $-0.2433^{*}$ & 0.1346 \\
\hline & G & & & & & & 1.0000 & -0.8610 & 0.5083 & -0.1861 & 0.3299 & -1.2691 & -0.0032 & -0.2448 & 0.1989 \\
\hline \multirow[t]{2}{*}{ Specific leaf weight $\left(\mathrm{g} \mathrm{cm}^{-2}\right)$ at 40 DAS } & $P$ & & & & & & & 1.0000 & $0.3037^{* \star}$ & 0.1287 & 0.1485 & 0.0434 & $-0.2177^{*}$ & $0.2445^{\star}$ & -0.1669 \\
\hline & G & & & & & & & 1.0000 & -0.7990 & 0.2373 & 0.3613 & -0.0069 & -0.4499 & 0.4906 & -0.5425 \\
\hline \multirow[t]{2}{*}{ Specific leaf weight $\left(\mathrm{g} \mathrm{cm}^{-2}\right)$ at 60 DAS } & $P$ & & & & & & & & 1.0000 & -0.0310 & $0.1961^{*}$ & 0.1799 & -0.0411 & 0.1632 & -0.0122 \\
\hline & G & & & & & & & & 1.0000 & 0.0317 & -0.6597 & -3.1945 & 0.1581 & -0.4759 & 0.1111 \\
\hline \multirow[t]{2}{*}{ Relative water content (\%) at 40 DAS } & $P$ & & & & & & & & & 1.0000 & 0.0972 & $-0.2262^{*}$ & 0.0924 & $0.3324^{* *}$ & 0.0488 \\
\hline & G & & & & & & & & & 1.0000 & 0.1109 & -2.1358 & 0.1071 & 0.3636 & -0.1525 \\
\hline \multirow[t]{2}{*}{ Relative water content (\%) at 60 DAS } & $P$ & & & & & & & & & & 1.0000 & 0.0619 & $-0.3257^{* *}$ & $0.2093^{*}$ & -0.1013 \\
\hline & G & & & & & & & & & & 1.0000 & 0.5043 & -0.3458 & 0.2231 & -0.1002 \\
\hline \multirow[t]{2}{*}{ Root length(cm) } & $P$ & & & & & & & & & & & 1.0000 & $-0.3406^{* *}$ & $0.2531^{*}$ & -0.0490 \\
\hline & G & & & & & & & & & & & 1.0000 & -2.1434 & 1.7166 & -0.2182 \\
\hline \multirow[t]{2}{*}{ Dry haulm weight per plant (g) } & $P$ & & & & & & & & & & & & 1.0000 & $-0.5574^{* *}$ & 0.0964 \\
\hline & G & & & & & & & & & & & & 1.0000 & -0.5616 & 0.1302 \\
\hline \multirow[t]{2}{*}{ Harvest index (\%) } & $P$ & & & & & & & & & & & & & 1.0000 & $0.3198^{*}$ \\
\hline & G & & & & & & & & & & & & & 1.0000 & -0.1723 \\
\hline
\end{tabular}

* Significant at $5 \%$ level; ** Significant at $1 \%$ level. 
Table 2. Path coefficients for water use efficiency traits and pod yield in groundnut.

\begin{tabular}{|c|c|c|c|c|c|c|c|c|c|c|c|c|c|c|c|}
\hline \multirow{2}{*}{ Character } & & \multirow{2}{*}{$\begin{array}{l}\text { Days to } \\
\mathbf{5 0 \%} \\
\text { flowering }\end{array}$} & \multirow{2}{*}{$\begin{array}{l}\text { Days to } \\
\text { maturity }\end{array}$} & \multicolumn{2}{|c|}{$\begin{array}{l}\text { SPAD chlorophyll } \\
\text { meter reading at }\end{array}$} & \multicolumn{2}{|c|}{$\begin{array}{l}\text { Specific leaf area } \\
\left(\mathrm{cm}^{2} \mathrm{~g}^{-1}\right) \text { at }\end{array}$} & \multicolumn{2}{|c|}{$\begin{array}{l}\text { Specific leaf weight } \\
\left(\mathrm{g} \mathrm{cm}^{-2}\right) \text { at }\end{array}$} & \multicolumn{2}{|c|}{$\begin{array}{l}\text { Relative water content } \\
(\%) \text { at }\end{array}$} & \multirow{2}{*}{$\begin{array}{l}\text { Root } \\
\text { length } \\
\text { (cm) }\end{array}$} & \multirow{2}{*}{$\begin{array}{l}\text { Dry haulm } \\
\text { weight per } \\
\text { plant (g) }\end{array}$} & \multirow{2}{*}{$\begin{array}{l}\text { Harvest } \\
\text { index (\%) }\end{array}$} & \multirow{2}{*}{$\begin{array}{l}\text { Pod yield } \\
\text { per plant }(g)\end{array}$} \\
\hline & & & & $\begin{array}{l}40 \\
\text { DAS }\end{array}$ & 60 DAS & 40 DAS & 60 DAS & 40 DAS & 60 DAS & 40 DAS & 60 DAS & & & & \\
\hline \multirow{2}{*}{ Days to $50 \%$ flowering } & $P$ & -0.2810 & -0.0680 & -0.0617 & -0.0787 & -0.0013 & 0.0200 & -0.0045 & -0.0711 & -0.0022 & 0.0271 & 0.0211 & -0.0515 & -0.0250 & -0.2070 \\
\hline & G & -0.0809 & -0.0978 & -0.0199 & -0.0272 & -0.0006 & 0.0070 & -0.0104 & 0.0417 & -0.0028 & 0.0084 & 0.0379 & -0.0162 & -0.0079 & -0.3697 \\
\hline \multirow{2}{*}{ Days to maturity } & $P$ & 0.0818 & 0.3381 & 0.0487 & 0.0341 & 0.0607 & -0.0473 & -0.0656 & 0.0374 & 0.2412 & 0.0413 & -0.0054 & 0.0277 & 0.0868 & 0.0699 \\
\hline & G & -0.0582 & -0.0481 & -0.0171 & -0.0267 & -0.0393 & 0.0299 & 0.1097 & -0.0165 & -0.1648 & -0.0340 & -0.0235 & -0.0162 & -0.0079 & 0.5758 \\
\hline \multirow{2}{*}{ SPAD chlorophyll meter reading at $40 \mathrm{DAS}$} & $P$ & 0.0703 & 0.0461 & 0.3200 & 0.1263 & 0.0490 & -0.0517 & -0.0547 & -0.0032 & 0.0064 & -0.0452 & -0.0180 & 0.1407 & -0.0228 & 0.0296 \\
\hline & G & 0.1019 & 0.1470 & 0.4142 & 0.1776 & 0.0670 & -0.0691 & -0.1423 & 0.0586 & -0.0022 & -0.0649 & -0.3582 & 0.1896 & -0.0300 & 0.0181 \\
\hline \multirow{2}{*}{ SPAD chlorophyll meter reading at $60 \mathrm{DAS}$} & $P$ & -0.0700 & -0.0252 & -0.0986 & -0.2498 & -0.0230 & 0.0352 & 0.0059 & -0.0329 & 0.0222 & 0.0809 & -0.0410 & -0.0109 & -0.0319 & $0.2391^{*}$ \\
\hline & G & -0.1050 & -0.1730 & -0.1339 & -0.3124 & -0.0290 & 0.0460 & 0.0255 & 0.1910 & 0.0337 & 0.1160 & -0.2394 & -0.0154 & -0.0431 & -0.2132 \\
\hline \multirow{2}{*}{ Specific leaf area $\left(\mathrm{cm}^{2} \mathrm{~g}^{-1}\right)$ at 40 DAS } & $P$ & -0.0014 & -0.0542 & -0.0462 & -0.0278 & -0.3019 & -0.0465 & 0.2298 & 0.0731 & 0.0378 & 0.0567 & 0.0113 & -0.0348 & 0.0552 & -0.1168 \\
\hline & G & -0.0017 & -0.1762 & -0.0349 & -0.0201 & -0.2159 & -0.0341 & 0.3417 & -0.1517 & 0.0292 & 0.0444 & 0.0523 & -0.0251 & 0.0400 & -0.1622 \\
\hline \multirow{2}{*}{ Specific leaf area $\left(\mathrm{cm}^{2} \mathrm{~g}^{-1}\right)$ at 60 DAS } & $P$ & -0.0153 & -0.0301 & -0.0348 & -0.0303 & 0.0332 & 0.2151 & -0.0905 & -0.0367 & -0.0365 & 0.0660 & -0.0406 & -0.0007 & -0.0523 & 0.1346 \\
\hline & G & -0.0411 & -0.2964 & -0.0796 & -0.0702 & 0.0753 & 0.4770 & -0.4107 & 0.2425 & -0.0888 & 0.1574 & -0.6054 & -0.0015 & -0.1168 & 0.1989 \\
\hline \multirow{2}{*}{ Specific leaf weight $\left(\mathrm{g} \mathrm{cm}^{-2}\right)$ at 40 DAS } & $P$ & 0.0006 & -0.0067 & -0.0059 & -0.0008 & -0.0262 & -0.0145 & 0.0344 & 0.0104 & 0.0044 & 0.0051 & 0.0015 & -0.0075 & 0.0084 & -0.1669 \\
\hline & G & 0.0047 & -0.0837 & -0.0126 & -0.0030 & -0.0581 & -0.0316 & 0.0367 & -0.0293 & 0.0087 & 0.0133 & -0.0003 & -0.0165 & 0.0180 & -0.5425 \\
\hline \multirow{2}{*}{ Specific leaf weight $\left(\mathrm{g} \mathrm{cm}^{-2}\right)$ at 60 DAS } & $P$ & 0.0205 & 0.0090 & -0.0008 & 0.0107 & -0.0196 & -0.0138 & 0.0246 & 0.0811 & -0.0025 & 0.0159 & 0.0146 & -0.0033 & 0.0132 & -0.0122 \\
\hline & G & -0.0022 & 0.0014 & 0.0006 & -0.0026 & 0.0029 & 0.0021 & -0.0033 & 0.0042 & 0.0001 & -0.0028 & -0.0134 & 0.0007 & -0.0020 & 0.1111 \\
\hline \multirow{2}{*}{ Relative water content (\%) at $40 \mathrm{DAS}$} & $P$ & -0.0026 & -0.2404 & -0.0067 & 0.0300 & 0.0422 & 0.0572 & -0.0434 & 0.0104 & -0.3370 & -0.0328 & 0.0762 & -0.0311 & -0.1120 & 0.0488 \\
\hline & G & 0.0018 & 0.1747 & -0.0003 & -0.0055 & -0.0069 & -0.0095 & 0.0121 & 0.0016 & 0.0510 & 0.0057 & -0.1090 & 0.0055 & 0.0186 & -0.1525 \\
\hline \multirow{2}{*}{ Relative water content (\%) at 60 DAS } & $P$ & 0.0326 & -0.0412 & 0.0476 & 0.1094 & 0.0634 & -0.1036 & -0.0501 & -0.0662 & -0.0328 & -0.3375 & -0.0209 & 0.1099 & -0.0706 & -0.1013 \\
\hline & G & 0.0338 & -0.2306 & 0.0512 & 0.1213 & 0.0672 & -0.1078 & -0.1181 & 0.2156 & -0.0362 & -0.3269 & -0.1648 & 0.1130 & -0.0729 & -0.1002 \\
\hline \multirow{2}{*}{ Root length(cm) } & $\mathrm{P}$ & 0.0238 & 0.0050 & 0.0177 & -0.0518 & 0.0118 & 0.0596 & -0.0137 & -0.0568 & 0.0715 & -0.0196 & -0.3160 & 0.1076 & -0.0800 & -0.0490 \\
\hline & $G$ & -0.0129 & 0.0134 & -0.0238 & 0.0211 & -0.0067 & -0.0350 & -0.0002 & -0.0881 & -0.0589 & 0.0139 & 0.0276 & -0.0591 & 0.0473 & -0.2182 \\
\hline \multirow{2}{*}{ Dry haulm weight per plant (g) } & $P$ & -0.1722 & -0.0769 & -0.4130 & -0.0409 & -0.1081 & 0.0031 & 0.2044 & 0.0386 & -0.0867 & 0.3058 & 0.3198 & -0.9390 & 0.5234 & 0.0964 \\
\hline & G & -0.3340 & -0.7020 & -0.7656 & -0.0824 & -0.1946 & 0.0054 & 0.7523 & -0.2644 & -0.1791 & 0.5782 & 3.5839 & -1.6721 & 0.9391 & 0.1302 \\
\hline \multirow{2}{*}{ Harvest index (\%) } & $P$ & -0.0415 & -0.1195 & 0.0332 & -0.0593 & 0.0851 & 0.1132 & -0.1138 & -0.0759 & -0.1547 & -0.0974 & -0.1178 & 0.2594 & -0.4654 & $0.3198^{*}$ \\
\hline & $G$ & -0.1098 & -1.3943 & 0.0813 & -0.1549 & 0.2080 & 0.2744 & -0.5501 & 0.5336 & -0.4077 & -0.2501 & -1.9246 & 0.6297 & -1.1212 & -0.1723 \\
\hline $\begin{array}{l}\text { PHENOTYPIC RESIDUAL EFFECT = } \\
\text { GENOTYPIC RESIDUAL EFFECT = }\end{array}$ & & $\begin{array}{l}0.5590 \\
1.1860\end{array}$ & & & & & & & & & & & & & \\
\hline
\end{tabular}

${ }^{*}$ Significant at $5 \%$ level; ** Significant at $1 \%$ level; Diagonal values (Bold) : Direct effects. 
The maximum positive direct positive effect on pod yield per plant was contributed by days to maturity, SPAD chlorophyll meter reading at 40 days after sowing and 60 days after sowing, specific leaf area at 60 days after sowing, specific leaf area at 40 days after sowing and 60 days after sowing, dry haulms yield per plant and harvest index. Similar results were reported by Suneetha et al. (2004), Golakia et al. (2005), Korat et al. (2010), John et al. (2011) and John et al. (2013).

Days to 50 per cent flowering showed positive indirect effect through specific leaf area at 60 days after sowing, relative water content at 60 days after sowing and root length, while through days to maturity, SPAD chlorophyll meter reading at 40 days after sowing and 60 days after sowing, specific leaf area at 40 days after sowing, specific leaf weight at 40 days after sowing and 60 days after sowing, relative water content at 40 days after sowing, dry haulms yield per plant, harvest index and pod yield per plant showed negative indirect effect. Days to maturity showed positive indirect effect through days to 50 per cent flowering, SPAD chlorophyll meter reading at 40 days after sowing and 60 days after sowing, specific leaf area at 40 days after sowing, specific leaf weight at 60 days after sowing, relative water content at 40 days after sowing and 60 days after sowing, dry haulms yield per plant, harvest index and pod yield per plant. But negative indirect effect through specific leaf area at 60 days after sowing, specific leaf weight at 40 days after sowing and root length.

The character, SPAD chlorophyll meter reading at 40 days after sowing had positive indirect effect through days to 50 per cent flowering, days to maturity, SPAD chlorophyll meter reading at 60 days after sowing, specific leaf area at 40 days after sowing, specific leaf weight at 60 days after sowing, dry haulms yield per plant and pod yield per plant and negative indirect effect through specific leaf area at 60 days after sowing, specific leaf weight at 40 days after sowing, relative water content at 40 days after sowing and 60 days after sowing, root length and harvest index.

SPAD chlorophyll meter reading at 60 days after sowing had positive indirect effect through specific leaf area at 60 days after sowing, specific leaf weight at 40 days after sowing, relative water content at 40 days after sowing and 60 days after sowing, root length and harvest index. It had negative indirect effect through days to 50 per cent flowering, days to maturity, SPAD chlorophyll meter reading at 40 days after sowing, specific leaf area at 40 days after sowing, specific leaf weight at 60 days after sowing, root length, dry haulms yield per plant and harvest index.

Specific leaf area at 40 days after sowing had positive indirect effect through specific leaf weight at 40 days after sowing and 60 days after sowing, relative water content at 40 days after sowing and 60 days after sowing, root length and harvest index negative indirect effect through days to 50 per cent flowering, days to maturity, SPAD chlorophyll meter reading at 40 days after sowing and 60 days after sowing, specific leaf area at 60 days after sowing, dry haulms yield per plant and pod yield per plant. Specific leaf area at 60 days after sowing had positive indirect effect through SPAD chlorophyll meter reading at 40 days after sowing and 60 days after sowing relative water content at 60 days after sowing and pod yield per plant, while through days to 50 per cent flowering, days to maturity, SPAD chlorophyll meter reading at 40 days after sowing and 60 days after sowing, specific leaf weight at 40 days after sowing and 60 days after sowing, relative water content at 40 days after sowing, root length, dry haulms yield per plant and harvest index had shown indirect negative effect.

Specific leaf weight at 40 days after sowing had positive indirect effect through days to 50 per cent flowering, days to maturity, specific leaf weight at 60 days after sowing, relative water content at 40 days after sowing and 60 days after sowing, root length and harvest index and indirect negative effect was observed through shown SPAD chlorophyll meter reading at 40 days after sowing and 60 days after sowing, specific leaf area at 40 days after sowing and 60 days after sowing, dry haulms yield per plant and pod yield per plant. The character, specific leaf weight at 60 days after sowing had positive indirect effect through days to 50 per cent flowering, days to maturity, SPAD chlorophyll meter reading at 40 days after sowing, specific leaf weight at 40 days after sowing, relative water content at 60 days after sowing, dry haulms yield per plant and harvest index and indirect negative effect through SPAD chlorophyll meter reading at 40 days after sowing, specific leaf area at 40 days after sowing and 60 days after sowing, relative water content at 40 days after sowing, dry haulms yield per plant and pod yield per plant.

Relative water content at 40 days after sowing had positive indirect effect through SPAD chlorophyll meter reading at 60 days after sowing, specific leaf area at 40 days after sowing and 60 days after sowing, specific leaf weight at 60 days after sowing, root length and pod yield per plant. It had shown indirect negative effect through 
days to 50 per cent flowering, days to maturity, SPAD chlorophyll meter reading at 40 days after sowing, specific leaf weight at 40 days after sowing, relative water content at 60 days after sowing, dry haulms yield per plant and harvest index. Relative water content at 60 days after sowing had positive indirect effect through days to 50 per cent flowering, SPAD chlorophyll meter reading at 40 days after sowing and 60 days after sowing, specific leaf area at 40 days after sowing and dry haulms yield per plant and indirect negative effect through days to maturity, specific leaf area at 60 days after sowing, specific leaf weight at 40 days after sowing and 60 days after sowing, relative water content at 40 days after sowing, root length, harvest index and pod yield per plant.

The character, root length had positive indirect effect through days to 50 per cent flowering, days to maturity, SPAD chlorophyll meter reading at 40 days after sowing, specific leaf area at 40 days after sowing and 60 days after sowing, relative water content at 40 days after sowing and dry haulms yield per plant. While through SPAD chlorophyll meter reading at 60 days after sowing, specific leaf weight at 40 days after sowing and 60 days after sowing, relative water content at 60 days after sowing, harvest index and pod yield per plant had shown indirect negative effect.

The indirect positive effect was observed for dry haulms yield per plant through SPAD chlorophyll meter reading at 40 days after sowing, specific leaf weight at 40 days after sowing and 60 days after sowing, relative water content at 60 days after sowing, root length, harvest index and pod yield per plant and indirect negative effect through days to 50 per cent flowering, days to maturity, SPAD chlorophyll meter reading at 40 days after sowing, specific leaf area at 40 days after sowing and relative water content at 40 days after sowing. Harvest index had positive indirect effect through SPAD chlorophyll meter reading at 40 days after sowing, specific leaf area at 40 days after sowing and 60 days after sowing and dry haulms yield per plant. While through days to 50 per cent flowering, days to maturity, SPAD chlorophyll meter reading at 60 days after sowing, specific leaf weight at 40 days after sowing and 60 days after sowing, relative water content at 40 days after sowing and 60 days after sowing, root length and pod yield per plant had shown indirect negative effect.

From the above discussion on path coefficient analysis it can be concluded that, the maximum positive direct positive effect on pod yield per plant was contributed by days to maturity, SPAD chlorophyll meter reading at 40 days after sowing and 60 days after sowing, specific leaf area at 60 days after sowing, specific leaf area at 40 days after sowing and 60 days after sowing, dry haulms yield per plant and harvest indicating that these traits are the important yield contributing characters.

\section{Conflict of interest statement}

Authors declare that they have no conflict of interest.

\section{Acknowledgement}

This research was supported under the UGC Research award to the first author by the University Grants Commission (UGC), New Delhi is gratefully acknowledged.

\section{References}

Al-Jibouri, H. A., Miller, P. A., Robinson, H. F., 1958. Genotypic and environmental variance and covariance in an upland cotton cross of inter-specific origin. Agron. J. 50, 633-637.

Araus, J. L., Sauque, V., Malas, Serret, M. D., 1989. Seasonal changes in the photosynthetic capacity and leaf structure of Fatsia japonilla leaves grown in the shade house. J. Hortic. Sci. 64, 189-198.

Dewey, D. R., Lu, K. H., 1959. A correlation and path analysis of components of crested wheat grass seed production. Agron. J. 51, 515-518.

Golakia, P.R., Makne, V.G., Monpara, B.A., 2005. Heritable variation and association in Virginia and Spanish bunch group of groundnut. Nat. J. Plant Improv. 7, 50-53.

John, K., Raghava Reddy, P., Hariprasad Reddy, K., Sudhakar, P., Eswar Reddy, N.P., 2011. Character association and path coefficient analysis among physiological (water use efficiency) traits and yield in $\mathrm{F}_{2}$ population of groundnut crosses (Arachis hypoaea L.). Plant Arch. 11 (2), 11231128 .

John, K., Raghava Reddy, P., Hariprasad Reddy, K., Sudhakar, P., Eswar Reddy, N.P., 2012. Variability, correlation and path coefficient analysis for morpho-physiological traits in groundnut (Arachis hypogaea L.). Plant Arch. 12(1), 315325.

John, K., Raghava Reddy, P., Hariprasad Reddy, K., Sudhakar, P., Eswar Reddy, N.P., 2013. Character association and path coefficient analysis for morpho-physiological traits in groundnut (Arachis hypogaea L.). Andhra Agric. J. 60(2), 283-291.

Korat, V. P., Pithia, M. S., Savaliya, J. J., Pansuriya, A. G., Sodavadiya, P. R., 2010. Studies on characters association and path analysis for seed yield and its components in groundnut (Arachis hypogaea L.). Legume Res. 33(3), 211-216. 
Nageswara Rao, R. C., Wright, G. C., 1994. Stability of the relationship between specific leaf area and carbon isotope discrimination across environments in peanut. Crop Sci. 34, 98-103.

Suneetha, K., Dasarada Rami Reddy, C., Ramana, J. V., 2004. Genetic variability and character association in groundnut. Short Papers Presented at the National Symposium On
Enhancing Productivity of Groundnut for Sustaining Food and Nutritional Security. 11-13 October-2004 at NRCG, Junagadh.

Vaithiyalingan, M., Manohara, V., Ramamoorthi, N., 2010. Association analysis among the yield and yield attributes of early season drought tolerant groundnut (Arachis hypogaea L.). Electronic J. Plant Breed. I(5), 1347-1350.

\section{How to cite this article:}

John, K., Raghava Reddy, P., 2016. Studies on characters association and path coefficient analysis for yield and water use efficiency traits in groundnut (Arachis hypogaea L.). Int. J. Curr. Res. Biosci. Plant Biol. 3(10), 128-135. doi: http://dx.doi.org/10.20546/ijcrbp.2016.310.015 